\title{
The Instabilities of Periodic Traveling Water Waves with Respect to Transverse Perturbations
}

\author{
Katie Oliveras and Bernard Deconinck \\ ABSTRACT. We show that the exact reformulation of the classical surface water \\ wave problem due to Ablowitz, Fokas and Musslimani, provides a convenient \\ framework to investigate the instabilities of one-dimensional stationary peri- \\ odic waves, with respect to transverse perturbations. Such perturbations have \\ trigonometric dependence on the transverse variable, and are bounded (typi- \\ cally quasi periodic) in the longitudinal direction. Using the new formulation, \\ we examine waves in both deep and shallow water, confirming previous results \\ about their instabilities due to McLean and Francius \& Kharif, among others.
}

\section{Introduction}

The objective of this paper is to examine the stability of one-dimensional periodic surface gravity waves with respect to two-dimensional perturbations using a recent nonlocal reformulation of the water wave problem due to Ablowitz, Fokas and Musslimani AFM06. Of course, there exists a great wealth of literature on the stability of traveling water waves, and by no means can this be completely covered, nor replicated, within the context of this paper. We discuss the major results that are fundamental to our investigation.

Perhaps the earliest results on the stability of surface water waves are those discussing the instability of small-amplitude waves in deep water with respect to long-wave perturbations. This modulational (or Benjamin-Feir) instability was discovered in 1967 by Benjamin Ben67, Benjamin \& Feir BF67 and Whitham Whi67. Benjamin Ben67 considered a Stokes wave solution perturbed by two sidebands. By examining the Fourier mode expansion of the perturbed solution, he determined that waves of period $L$ in water of dimensionless depth $k h>1.363$ $(k=2 \pi / L)$, are unstable with respect to long-wave perturbations.

Since the 1960s, researchers have been using numerical techniques to determine the stability properties of traveling waves due to the complicated nature of the underlying equations of motion. Examples of in-depth numerical studies on one-dimensional solutions perturbed by one-dimensional disturbances include the early work of Longuet-Higgins LH78a, LH78b, and the current work of Nicholls Nic09. Recently, we have examined the spectral stability of periodic

2010 Mathematics Subject Classification. Primary 54C40, 14E20; Secondary 46E25, 20C20.

Key words and phrases. Fluid flow, nonlinear waves, stability.

The first author was supported in part by NSF Grant NSF-DMS-VIGRE-0354131.

NSF-DMS-0604546. 
traveling waves in the one-dimensional setting DO. In addition to recovering expected results (i.e. waves in deep water are modulationally unstable with respect to long-wave perturbations), we found there are specific classes of quasi-periodic perturbations that yield instabilities in shallow water, even for small-amplitude waves.

As stated, the above results are limited to one-dimensional periodic traveling waves perturbed by one-dimensional periodic or quasi-periodic disturbances. As an intermediate step between investigating the one-dimensional and the twodimensional problem, different researchers have considered the effects of two- dimensional perturbations on one-dimensional traveling wave solutions. In other words, they have investigated the transverse instabilities of the one-dimensional solutions. Early notable work on the transverse instabilities of solutions of the Euler equations was conducted by Bryant Bry78. Using a set of truncated nonlinear approximations to the Euler equations for water of finite depth, he explored the instabilities using a small number of wave interactions. Several years later, McLean presented the first results on the transverse instabilities of one-dimensional solutions to the Euler equations for water of finite depth. McLean et al. (Refs. [McL82a, McL82b, $\mathbf{M M M}^{+}$81] ) considered perturbations of the form

$$
\begin{gathered}
\eta_{1}=e^{-\sigma t} e^{i p x+i q y} \sum_{j=-\infty}^{\infty} a_{j} e^{i j x}, \text { and } \\
\phi_{1}=e^{-\sigma t} e^{i p x+i q y} \sum_{j=-\infty}^{\infty} b_{j} e^{i j x} \frac{\cosh \left(\kappa_{j}(z+h)\right)}{\cosh \left(\kappa_{j} h\right)} .
\end{gathered}
$$

Here $\eta_{1}$ and $\phi_{1}$ are the perturbations of the surface elevation and the velocity potential in the interior of the fluid, respectively. Substituting these expansions into the original equations of motion, McLean calculated the growth rates $-\sigma$ associated with the linearized problem for waves in deep water. He found that in deep water, small-amplitude one-dimensional waves are most unstable to one-dimensional perturbations. However, as the amplitude of the wave is increased, the wave becomes more unstable with respect to two-dimensional perturbations. More recently, McLean's stability calculations have been extended to waves of even greater amplitude by Kharif \& Ramamonjiarisoa[KR90 and to waves in water of shallower depths by Francius \& Kharif[FK06].

The purpose of this paper is to demonstrate that we can replicate these transverse instability results using the nonlocal formulation of Ablowitz, Fokas, and Musslimani [AFM06] with less computational effort. In order to numerically calculate the spectral instabilities, we must determine (i) one-dimensional traveling wave solutions, and (ii) the growth rates associated with two-dimensional perturbations of these one-dimensional traveling waves. We have recently shown that one-dimensional periodic traveling waves solutions can be determined by numerically solving the single equation

$$
\int_{0}^{L} e^{-i k x} \sqrt{\left(1+\eta_{x}^{2}\right)\left(c^{2}-2 g \eta\right)} \sinh (k(\eta+h)) d x=0
$$

$\forall k \in \frac{2 \pi}{L} \mathbb{Z}_{0}$, for the surface elevation $\eta(x)$ moving with wave-speed $c$, see Ref. [DO]. Using the numerical scheme outlined in Deconinck \& Oliveras DO, we determine one-dimensional traveling wave solutions for various wave amplitudes and 
water depths. These one-dimensional solutions are perturbed with two-dimensional (quasi-) periodic disturbances. Substituting the two-dimensional perturbations into the equations of motion, we calculate the linear growth rates using a Fourier-Floquet decomposition, see for example Ref. [DK06]. We find that our stability results are in general agreement with those of Francius \& Kharif [FK06] and the other sources mentioned above. Although a direct comparison is not possible (see Section 5), it appears that our method produces more accurate results at a significantly reduced computational cost.

\section{Equations of Motion}

We consider Euler's equations describing the dynamics of the surface of an ideal fluid in three dimensions. For the periodic problem, this requires us to solve Laplace's equation inside the fluid domain $D$ with periodic boundary conditions in both horizontal directions $x$ and $y$. The fluid domain $D$ is shown in Figure 1. The equations of motion are

$$
\begin{array}{lr}
\phi_{x x}+\phi_{y y}+\phi_{z z}=0, & (x, y, z) \in D, \\
\phi_{z}=0, & z=-h, \\
\eta_{t}+\phi_{x} \eta_{x}+\phi_{y} \eta_{y}=\phi_{z}, & z=\eta(x, y, t),
\end{array}
$$

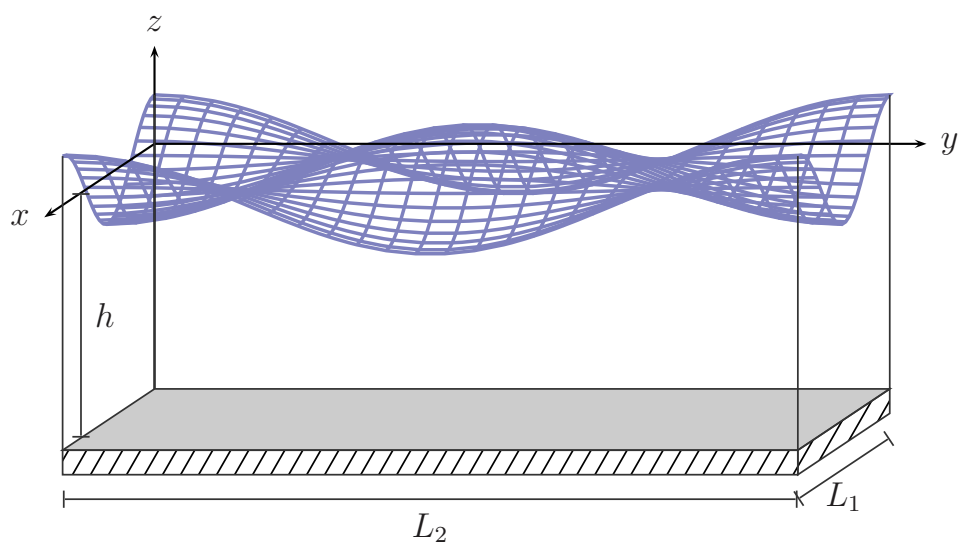

FIgURE 1. The fluid domain for a water wave surface with periodic boundary conditions. 


$$
\phi_{t}+\frac{1}{2}\left(\phi_{x}^{2}+\phi_{y}^{2}\right)+g \eta=0, \quad z=\eta(x, y, t),
$$

where $\phi=\phi(x, y, z, t)$ is the velocity potential, $\eta=\eta(x, y, t)$ is the surface elevation, $g$ is the acceleration of gravity, and $h$ is the constant depth of the fluid when at a state of rest. In this paper, we ignore the effects of surface tension. We require periodic boundary conditions with period $L_{1}$ in the $x$-direction and $L_{2}$ in the $y$ direction. This provides additional boundary conditions on the surface elevation as well as on the velocity potential once the induced mean flow has been eliminated. We have assumed finite depth in the above formulation. The formulation is also valid in infinite depth.

As discussed in Ref. [DO, Euler's equations as written above are challenging to work with directly: they are a free-boundary problem with nonlinear boundary conditions specified at the unknown boundary. For the two-dimensional problem (i.e., one-dimensional surface), there are several reformulations that reduce these complications. However for the three-dimensional problem (with two-dimensional surface), there are fewer alternative formulations. One of the commonly used reformulations is that due to Zakharov, Craig and Sulem Zak68, CS93. A minor disadvantage to the Zakharov-Craig-Sulem formulation is that for numerical implementations, one must truncate the series expansion of the Dirichlet-to-Neumann operator. The nonlocal formulation presented below does not require such a truncation.

Ablowitz, Fokas and Musslimani (AFM) AFM06 introduced a new, nonlocal reformulation of the Euler equations, valid for surface waves localized on the whole line or the whole plane. It is essentially trivial to extend this formulation to periodic boundary conditions. In a traveling coordinate frame where $\mathbf{x}=(x, y)$ is moving with speed $\bar{c}=\left(c_{x}, c_{y}\right)$, the equations of motion can be written as

$$
\begin{gathered}
\int_{0}^{L_{2}} \int_{0}^{L_{1}} e^{-i \bar{k} \cdot \mathbf{x}}\left(i\left(\eta_{t}-\bar{c} \cdot \nabla \eta\right) \cosh (\kappa(h+\eta))\right. \\
\left.-\frac{\bar{k} \cdot \nabla q}{\kappa} \sinh (\kappa(h+\eta))\right) d x d y=0, \\
q_{t}-\bar{c} \cdot \nabla q+\frac{1}{2}|\nabla q|^{2}+g \eta-\frac{1}{2} \cdot \frac{\left(\eta_{t}+\nabla q \cdot \nabla \eta\right)^{2}}{1+|\nabla \eta|^{2}}=0,
\end{gathered}
$$

where $\nabla=\left(\partial_{x}, \partial_{y}\right), q(x, y, t)=\phi(x, y, \eta(x, y, t), t)$ is the velocity potential evaluated at the surface, $\bar{k}=\left(k_{x}, k_{y}\right), \kappa=\sqrt{k_{x}^{2}+k_{y}^{2}}$ and

$$
\Lambda=\left\{\bar{k} \in \mathbb{R}^{2} \mid k_{x}=\frac{2 m \pi}{L_{1}}, k_{y}=\frac{2 n \pi}{L_{2}},(m, n) \in \mathbb{Z}^{2}-\{(0,0)\}\right\} .
$$

For the remainder of this paper, we use this nonlocal formulation of the waterwave problem. We refer the reader to Refs. DO, AFM06, AH08 for additional information regarding this exact reformulation of the water-wave problem.

Since our goal is to determine the stability of one-dimensional periodic traveling waves with respect to two-dimensional perturbations, we must use the full equations for the two-dimensional surface gravity wave to examine stability. Since 
we only consider the stability of one-dimensional solutions, we reduce the equations of motion by eliminating the $y$-dependence in (2.5) and (2.6) to determine the traveling wave solutions moving in the $x$-direction with speed $c$. In Ref. [DO], we demonstrated that periodic one-dimensional traveling wave solutions are solutions of

$$
\int_{0}^{L} e^{-i k x} \sqrt{\left(1+\eta_{x}^{2}\right)\left(c^{2}-2 g \eta\right)} \sinh (k(\eta+h)) d x=0,
$$

$\forall k \in \frac{2 \pi}{L} \mathbb{Z}_{0}$, for the surface elevation $\eta(x)$. This equation provides solutions to the fully nonlinear problem, and is easily solved numerically. To determine the velocity potential $q(x)$ at the surface (which is needed when examining the stability of traveling waves), we use the relationship

$$
q_{x}=c \pm \sqrt{\left(1+\eta_{x}^{2}\right)\left(c^{2}-2 g \eta\right)} .
$$

where we work with the negative sign in order to enforce that $u-c<0$ within the fluid bulk as well as along the free surface for all but the limiting wave. One-dimensional solutions are computed using an iterative pseudo-continuation approach as outlined in Ref. [DO. All solutions were calculated using at least 64 Fourier Modes and a residual error $<10^{-14}$.

\section{Stability Formulation}

Before we proceed to investigate the stability of the traveling wave profiles, we discuss what perturbations we wish to allow. In this paper, we plan to investigate the effects of two-dimensional pertubations. It may appear natural to consider disturbances of the same period as the underlying stationary wave in the $x$ direction, and vary the period of the perturbation in the $y$ direction. However, we wish to work with a more general class of disturbances, namely those that are bounded on the whole real plane. Specifically, for some disturbance $U(x, y)$, we use the notation ||$U(x, y) \|<\infty$ to mean that $\sup _{\mathbb{R}^{2}}|U(x, y)|<\infty$ and that $U(x, y)$ is continuous for all $(x, y) \in \mathbb{R}^{2}$. It is important to realize that this class is the largest class of perturbations allowed by the physical problem at hand. Indeed, disturbances should be bounded and continuous functions, but there is no physical reason to restrict their spatial dependence to be periodic.

In order to investigate the stability of the traveling wave profiles with respect to such perturbations, it is necessary to reformulate the governing equations. Equation (2.6) is a local statement and does not require modification. However, the current incarnation of the nonlocal equation (2.5) applies specifically to waves of period $L_{1}$ in the $x$-direction and $L_{2}$ in the $y$-direction. Let $\langle\langle f\rangle\rangle$ represent the average value of a function $f(\mathbf{x}), f: \mathbb{R}^{2} \rightarrow \mathbb{R}$ by

$$
\langle\langle f\rangle\rangle=\lim _{|A| \rightarrow \infty} \frac{1}{|A|} \iint_{A} f(x, y) d A,
$$

where $A$ is a rectangle in $\mathbb{R}^{2}$ and $|A|$ denotes its area.

It is clear that if $f(x, y)$ is periodic in both $x$ and $y$ with period $L_{1}$ and $L_{2}$ respectively, then $\langle\langle f\rangle\rangle$ is well defined. However, this two-dimensional spatial average is defined for the larger class of so-called almost-periodic functions, which contains 
the set of quasi-periodic functions Boh47. This leads us to replace (2.5) with the more general nonlocal equation

$$
\left\langle\left\langle e^{-i \bar{k} \cdot \mathbf{x}}\left(i\left(\eta_{t}-\bar{c} \cdot \nabla \eta\right) \cosh (\kappa(h+\eta))-\frac{\bar{k} \cdot \nabla q}{\kappa} \sinh (\kappa(h+\eta))\right)\right\rangle\right\rangle=0
$$

which is valid for all $\bar{k} \in \mathbb{R}_{0}^{2}=\mathbb{R}^{2}-\{(0,0)\}$. In fact, if we were to consider perturbations of increasingly larger period, the set of $\bar{k}$ values to be considered in Equation (2.5) would approach a dense subset of the real plane.

Having generalized the dynamical equations to accommodate the perturbations we wish to consider, we briefly discuss the definition of spectral stability. A stationary solution of a nonlinear problem is spectrally stable if there are no exponentially growing modes of the corresponding linearized problem. To determine the spectral stability of the periodic traveling wave solutions, we start by considering a onedimensional traveling wave solution $\left(\eta_{0}(x-c t), q_{0}(x-c t), c\right)$, which solves equation (2.7). In the same traveling coordinate frame, we add a small perturbation in both the $x$ and $y$ direction of the form

$$
\begin{aligned}
q(x-c t, y, t) & =q_{0}(x-c t)+\epsilon q_{1}(x-c t, y, t)+\mathcal{O}\left(\epsilon^{2}\right), \\
\eta(x-c t, y, t) & =\eta_{0}(x-c t)+\epsilon \eta_{1}(x-c t, y, t)+\mathcal{O}\left(\epsilon^{2}\right),
\end{aligned}
$$

where $\epsilon$ is a small parameter. The perturbation is moving in a traveling reference frame with speed $\bar{c}=(c, 0)$. Our goal is to determine the time dependence of the perturbation in order to determine how the deviation from the unperturbed solution evolves.

We begin with the local equation (2.6). Substituting the above into Equation (2.6) and keeping only terms to order $\epsilon$, we obtain

$$
q_{1, t}-c q_{1, x}+q_{0, x} q_{1, x}+g \eta_{1}-f \cdot\left(\eta_{1, t}+\eta_{1, x}\left(q_{0, x}-c\right)+\eta_{0, x} q_{1, x}-f \cdot \eta_{0, x} \eta_{1, x}\right)=0,
$$

where

$$
f=\frac{\eta_{0, x}\left(q_{0, x}-c\right)}{1+\eta_{0, x}^{2}} .
$$

Since the problem does not depend on $t$ or $y$ explicitly (recall that the functions $\eta_{0}$ and $q_{0}$ are both independent of $y$ and $t$, and depend only on $x$ ), we can use separation of variables to decompose $\eta_{1}(x, y, t)$ and $q_{1}(x, y, t)$ as

$$
\begin{aligned}
& \eta_{1}(x, y, t)=e^{\lambda t} e^{i \rho y} \tilde{\eta_{1}}(x)+c . c ., \\
& q_{1}(x, y, t)=e^{\lambda t} e^{i \rho y} \tilde{q_{1}}(x)+c . c .,
\end{aligned}
$$

without loss of generality. Here $\rho$ is the transverse wave number of the perturbation. Substituting into (3.2), we have

$$
\lambda \tilde{q}_{1}-c \tilde{q}_{1, x}+q_{0, x} \tilde{q}_{1, x}+g \tilde{\eta}_{1}-f \cdot\left(\lambda \tilde{\eta}_{1}+\tilde{\eta}_{1, x}\left(q_{0, x}-c\right)+\eta_{0, x} \tilde{q}_{1, x}-\eta_{0, x} f \cdot \tilde{\eta}_{1, x}\right)=0,
$$

which is the same linearization as that obtained in (Ref. [DO]) for the local equation with respect to one-dimensional perturbations.

Now we turn our attention to the nonlocal equation. For the nonlocal equation, we substitute the perturbation expansion into (3.1). This gives

$$
\left\langle\left\langle e^{-i \bar{k} \cdot \mathbf{x}}\left(i \eta_{1, t} \mathcal{C}_{\kappa}-i c \eta_{0, x} \kappa \eta_{1} \mathcal{S}_{\kappa}-i c \eta_{1, x} \mathcal{C}_{\kappa}-\frac{1}{\kappa}\left(k_{x} q_{1, x}+k_{y} q_{1, y}\right) \mathcal{S}_{\kappa}-k_{x} q_{0, x} \eta_{1} \mathcal{C}_{\kappa}\right)\right\rangle\right\rangle=0,
$$


where

$$
\mathcal{S}_{\kappa}=\sinh \left(\kappa\left(\eta_{0}+h\right), \mathcal{C}_{\kappa}=\cosh \left(\kappa\left(\eta_{0}+h\right)\right)\right. \text {. }
$$

As before, since the problem does not depend on $y$ or $t$ explicitly, we use separation of variables to decompose $\eta_{1}(x, y, t)$ and $q_{1}(x, y, t)$ as in (3.3). Using this decomposition, we obtain

$$
\begin{aligned}
\left\langle\left\langle e^{-i \bar{k} \cdot \mathbf{x}} e^{i \rho y}\right.\right. & \left(i \tilde{\eta}_{1, t} \mathcal{C}_{\kappa}-i c \eta_{0, x} \kappa \tilde{\eta}_{1} \mathcal{S}_{\kappa}-i c \tilde{\eta}_{1, x} \mathcal{C}_{\kappa}\right. \\
& \left.\left.\left.-\frac{1}{\kappa}\left(k_{x} \tilde{q}_{1, x}+i k_{y} \rho \tilde{q}_{1}\right) \mathcal{S}_{\kappa}-k_{x} q_{0, x} \tilde{\eta}_{1} \mathcal{C}_{\kappa}\right)\right\rangle\right\rangle=0,
\end{aligned}
$$

Since our new integrand depends on $y$ only through $e^{i\left(\rho-k_{y}\right) y}$, we eliminate the integration with respect to $y$ completely. Calculating the average value along the $y$ direction results in

$$
\lim _{M \rightarrow \infty} \frac{1}{M} \int_{-M / 2}^{M / 2} e^{-i\left(k_{y}-\rho\right) y} d y=\lim _{M \rightarrow \infty} \frac{2}{M\left(k_{y}-\rho\right)} \sin \left(\frac{M\left(k_{y}-\rho\right)}{2}\right) .
$$

Taking the limit as $M \rightarrow \infty$, the contribution is identically zero if $k_{y} \neq \rho$. Otherwise, if $k_{y}=\rho$, the contribution from the integral is unity. Thus, the linearization of the nonlocal equation simplifies to

$$
\begin{array}{r}
\left\langlee ^ { - i k _ { x } x } \left( i \lambda \tilde{\eta}_{1} \mathcal{C}_{\kappa}-i c \tilde{\eta}_{1, x} \mathcal{C}_{\kappa}-\eta_{0, x} c \kappa \tilde{\eta}_{1} \mathcal{S}_{\kappa}\right.\right. \\
\left.-\frac{1}{\kappa}\left(k_{x} \tilde{q}_{1, x}+i \rho^{2} \tilde{q}_{1}\right) \mathcal{S}_{\kappa}-k q_{0, x} \tilde{\eta}_{1} \mathcal{C}_{\kappa}\right\rangle=0,
\end{array}
$$

where now $\kappa=\sqrt{k_{x}^{2}+\rho^{2}}$ and

$$
\langle f\rangle=\lim _{M \rightarrow \infty} \frac{1}{M} \int_{-M / 2}^{M / 2} f(x) d x .
$$

It should be noted that if we equate $\rho=0$, the above equation reduces to the expression for the one-dimensional surface case presented elsewhere DO.

Having linearized the equations about the traveling wave solution, we have the following generalized eigenvalue problem:

$$
\begin{array}{r}
\lambda \tilde{q}_{1}-c \tilde{q}_{1, x}+q_{0, x} \tilde{q}_{1, x}+g \tilde{\eta}_{1}-f \cdot\left(\lambda \tilde{\eta}_{1}\right. \\
\left.+\tilde{\eta}_{1, x}\left(q_{0, x}-c\right)+\eta_{0, x} \tilde{q}_{1, x}-\eta_{0, x} f \cdot \tilde{\eta}_{1, x}\right)=0 \\
\lambda\left\langle e^{-i k_{x} x}\left(i \tilde{\eta}_{1} \mathcal{C}_{\kappa}\right)\right\rangle=\left\langlee ^ { - i k _ { x } x } \left( i c \tilde{\eta}_{1, x} \mathcal{C}_{\kappa}+i \eta_{0, x} c \kappa \tilde{\eta}_{1} \mathcal{S}_{\kappa}\right.\right. \\
\left.\left.+\frac{1}{\kappa}\left(k_{x} \tilde{q}_{1, x}+i \rho^{2} \tilde{q}_{1}\right) \mathcal{S}_{\kappa}+k_{x} q_{0, x} \tilde{\eta}_{1} \mathcal{C}_{\kappa}\right)\right\rangle .
\end{array}
$$


Equations (3.6) and (3.7) are valid for $\left(k_{x}, \rho\right) \in \mathbb{R}^{2}-\{(0,0)\}$, and can be rewritten compactly as

$$
\lambda \mathcal{L}_{1} U(x)=\mathcal{L}_{2} U(x)
$$

where $\mathcal{L}_{1}$ and $\mathcal{L}_{1}$ are $2 \times 2$ matrices of linear operators that depend on $\rho$, and whose entries are easily read off from (3.6) and (3.7).

Since the time dependence of the perturbation depends exponentially on $\lambda$, we can determine information about the stability of the underlying traveling wave by determining the solutions of this generalized eigenvalue problem. If any solutions $U(x)$ exist for which the corresponding $\lambda$ has a positive real part, the linear approximation of the solution grows exponentially in time and the perturbed solution will diverge from the stationary solution in the linear approximation. With this intuition in mind, the concept of spectral stability, as applicable to the generalized eigenvalue problem (3.8) is defined as

Definition 3.1 (Spectral Stability). A one-dimensional periodic traveling wave solution $\left(\eta_{0}(x), q_{0}(x), c\right)$ of Equations 2.7 and 2.8 (and thus of the Euler equations) is spectrally stable with respect to two-dimensional perturbations parameterized by $\rho$ if no element of the spectrum for the generalized eigenvalue problem 3.8 has strictly positive real part.

In order to use this definition, we recall the definition of the spectrum of the generalized eigenvalue problem.

Definition 3.2 (Spectrum of the Generalized Eigenvalue Problem). The spectrum of the generalized Eigenvalue problem $\lambda \mathcal{L}_{1} U(x)=\mathcal{L}_{2} U(x)$ is given by

$$
\sigma\left(\mathcal{L}_{2}, \mathcal{L}_{1}\right)=\{\lambda \in \mathbb{C}:\|U(x)\|<\infty\},
$$

where $U(x)$ solves $\lambda \mathcal{L}_{1} U(x)=\mathcal{L}_{2} U(x)$.

In order to examine spectral stability or instability, we need to locate the spectrum of the generalized eigenvalue problem 3.8. By definition of the spectrum, we determine those values of $\lambda \in \mathbb{C}$ for which Equation 3.8 has bounded solutions. Since the coefficient functions of Equation (3.8) are periodic in $x$ with period $L_{1}$, we decompose the perturbations further using Floquet's Theorem, see for instance Ref. [DK06]. Thus, both $\tilde{q}_{1}(x)$ and $\tilde{\eta}_{1}(x)$ can be written as

$$
\tilde{q}_{1}(x)=e^{i \mu x} \bar{q}_{1}(x), \text { and } \tilde{\eta}_{1}(x)=e^{i \mu x} \bar{\eta}_{1}(x),
$$

where $\mu \in\left[-\pi / L_{1}, \pi / L_{1}\right)$ and $\bar{q}_{1}$ and $\bar{\eta}_{1}$ are $2 \pi$ periodic functions.

Substituting the Floquet decomposition into the eigenvalue problem yields

$$
\begin{aligned}
& \lambda\left(\tilde{q}_{1}-f \tilde{\eta}_{1}\right)= c\left(i \mu+\partial_{x}\right) \bar{q}_{1}-q_{0, x}\left(i \mu+\partial_{x}\right) \bar{q}_{1}-g \bar{\eta}_{1} \\
&+f\left(\left(q_{0, x}-c\right)\left(i \mu+\partial_{x}\right) \bar{\eta}_{1}+\eta_{0, x}\left(i \mu+\partial_{x}\right) \bar{q}_{1}-\eta_{0, x} f\left(i \mu+\partial_{x}\right) \bar{\eta}_{1}\right), \\
& \lambda\left\langle e^{i\left(\mu-k_{x}\right) x}\left(i \tilde{\eta}_{1} \mathcal{C}_{\kappa}\right)\right\rangle=\left\langle\left(e ^ { i ( \mu - k _ { x } ) x } \left( i c \mathcal{C}_{\kappa}\left(i \mu+\partial_{x}\right) \bar{\eta}_{1}+i \eta_{0, x} c \kappa \bar{\eta}_{1} \mathcal{S}_{\kappa}\right.\right.\right. \\
&\left.\left.\quad+\frac{1}{\kappa}\left(k_{x}\left(i \mu+\partial_{x}\right)+i \rho^{2}\right) \bar{q}_{1} \mathcal{S}_{\kappa}+k_{x} q_{0, x} \bar{\eta}_{1} \mathcal{C}_{\kappa}\right)\right\rangle=0 .
\end{aligned}
$$


We represent the $2 \pi$-periodic portion of the eigenfunctions $\bar{q}_{1}$ and $\bar{\eta}_{1}$ by their Fourier series. In other words,

$$
\bar{q}_{1}(x)=\sum_{m=-\infty}^{\infty} \hat{Q}_{m} e^{i m x}, \bar{\eta}_{1}(x)=\sum_{m=-\infty}^{\infty} \hat{N}_{m} e^{i m x}
$$

Additionally, we represent the periodic variable coefficient functions by their Fourier series as well. Substituting the series representations into the Floquet decomposition, we are able to determine a bi-infinite matrix eigenvalue problem, where the eigenvectors are the Fourier coefficients $\hat{Q}_{j}$ and $\hat{N}_{j}$ for $j \in\{0, \pm 1, \pm 2, \ldots\}$.

The Fourier decomposition proceeds in a straightforward manner for the local equation. Substituting the Fourier expansions into the local equation, we obtain

$$
\begin{array}{r}
\lambda\left(\sum_{m=-\infty}^{\infty} \hat{F}_{3, n-m} \hat{N}_{m}-\hat{Q}_{n}\right)=-g \hat{N}_{n}+\sum_{m=-\infty}^{\infty}(i \mu+i m) \hat{F}_{1, n-m} \hat{N}_{m} \\
+\sum_{m=-\infty}^{\infty} \hat{F}_{2, n-m}(i \mu+i m) \hat{Q}_{m},
\end{array}
$$

for $n \in \mathbb{Z}-\{0\}$, where the coefficients $\hat{F}_{j, n}$ are given by the following expressions

$$
\begin{aligned}
& \hat{F}_{1, n}=\int_{0}^{L_{1}} e^{-\frac{2 i n \pi x}{L_{1}}} f\left(\eta_{0}, q_{0}\right)\left(q_{0, x}-c-\eta_{0, x} f\left(\eta_{0}, q_{0}\right)\right) d x \\
& \hat{F}_{2, n}=\int_{0}^{L_{1}} e^{-\frac{2 i n \pi x}{L_{1}}}\left(q_{0, x}-c-\eta_{0, x} f\left(\eta_{0}, q_{0}\right)\right) d x \\
& \hat{F}_{3, n}=\int_{0}^{L_{1}} e^{-\frac{2 i n \pi x}{L_{1}}} f\left(\eta_{0}, q_{0}\right) d x
\end{aligned}
$$

For the nonlocal equation, we employ a similar strategy as used elsewhere $\mathbf{D O}$. There are two slight differences. First, the hyperbolic sine and cosine terms depend on $\kappa=\sqrt{k_{x}^{2}+\rho^{2}}$ instead of $k_{x}$ directly. Second, there is an additional term depending on the perturbation of the velocity potential and on $\rho$.

Substituting the Fourier series into the nonlocal equation and representing all variable coefficients by their Fourier series representation, we have

$$
\begin{aligned}
& {\left[\sum_{j=-\infty}^{\infty} \sum_{m=-\infty}^{\infty}\left(\hat{G}_{1, j}^{\left(k_{x}\right)}+i c(i \mu+i m) \hat{G}_{2, j}^{\left(k_{x}\right)}\right) \hat{N}_{m}\right.} \\
& \left.\quad+\sum_{j=-\infty}^{\infty} \sum_{m=-\infty}^{\infty}\left(\frac{k_{x}}{\kappa}(i \mu+i m)+\frac{i \rho^{2}}{\kappa}\right) \hat{G}_{3, j}^{\left(k_{x}\right)} \hat{Q}_{m}\right]\left\langle E_{j m}\right\rangle \\
& \quad=i \lambda \sum_{j=-\infty}^{\infty} \sum_{m=-\infty}^{\infty} \hat{G}_{2, j}^{\left(k_{x}\right)} \hat{N}_{m}\left\langle E_{j m}\right\rangle
\end{aligned}
$$


where

$$
\begin{aligned}
\hat{G}_{1, n}^{\left(k_{x}\right)} & =\int_{0}^{L_{1}} e^{-\frac{2 i n \pi x}{L_{1}}}\left(i \kappa c \eta_{0, x} \mathcal{S}_{\kappa}+k_{x} q_{0} \mathcal{C}_{\kappa}\right) d x \\
\hat{G}_{2, n}^{\left(k_{x}\right)} & =\int_{0}^{L_{1}} e^{-\frac{2 i n \pi x}{L_{1}}} \mathcal{C}_{\kappa} d x \\
\hat{G}_{3, n}^{\left(k_{x}\right)} & =\int_{0}^{L_{1}} e^{-\frac{2 i n \pi x}{L_{1}}} \mathcal{S}_{\kappa} d x
\end{aligned}
$$

Each term in the double infinite sum contains the average value of

$$
E_{j m}=\exp \left[i x\left(\mu-k_{x}+m+j\right)\right]
$$

This average value is identically zero unless the quantity $\mu-k_{x}+m+j$ is zero so that $\left\langle E_{j m}\right\rangle=1$. In other words the contribution from the integral is identically zero unless

$$
k_{x}=\mu+n,
$$

for some integer $n$. Thus, the only contribution in the integral equation occurs when $k_{x}$ is a shift of the original dual lattice. This allows us to collapse the double infinite sum to a single infinite sum given by

$$
\begin{gathered}
\sum_{m=-\infty}^{\infty}\left(\hat{G}_{1, n-m}^{(k(\mu))}+i c(i \mu+i m) \hat{G}_{2, n-m}^{(k(\mu))}\right) \hat{N}_{m} \\
\left.+\sum_{j=-\infty}^{\infty} \sum_{m=-\infty}^{\infty}(i \mu+i m) \hat{G}^{(k(\mu))}(\mu+n)_{3, n-m} \hat{Q}_{m}\right] \\
\quad=i \lambda \sum_{m=-\infty}^{\infty} \hat{G}_{2, n-m}^{(k(\mu))} \hat{N}_{m}
\end{gathered}
$$

where $k(\mu)=\mu+n$. The above equation holds for all $n \in \mathbb{Z}$.

Combining the above equation with (3.12) yields a generalized bi-infinite eigenvalue problem for the spectrum of the linearized operator about the stationary traveling wave solutions. We define the quantity $\hat{\mathbf{X}}$ as the bi-infinite vector of Fourier coefficients $\hat{Q}_{j}$ and $\hat{N}_{j}$ for all $j \in \mathbb{Z}: \hat{\mathbf{X}}=\left[\ldots, \hat{N}_{-1}, \hat{N}_{0}, \hat{N}_{1}, \ldots \hat{Q}_{-1}, \hat{Q}_{0}, \hat{Q}_{1}, \ldots\right]^{T}$. With this notation, we rewrite the generalized eigenvalue problem as

$$
\left[\begin{array}{cc}
A(\mu) & B(\mu) \\
C(\mu, \rho) & D(\mu, \rho)
\end{array}\right] \hat{\mathbf{X}}=\lambda\left[\begin{array}{cc}
-I & S(\mu) \\
0 & V(\mu, \rho)
\end{array}\right] \hat{\mathbf{X}}
$$




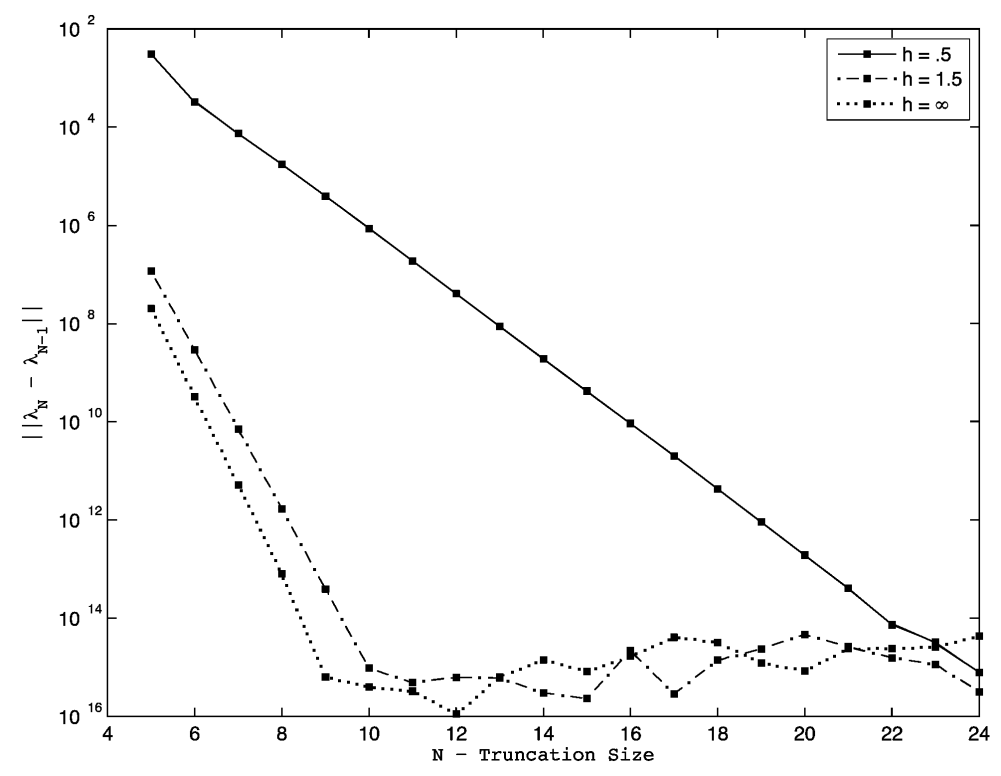

FiguRE 2. Cauchy error for the maximal eigenvalue corresponding to a solution with amplitude $a=.01$ in water of various depths.

where

$$
\begin{aligned}
A(\mu)_{n, m} & =-g \delta(n-m)+(i \mu+i m) \hat{F}_{1, n-m}, \\
B(\mu)_{n, m} & =\hat{F}_{2, n-m}(i \mu+i m), \\
C(\mu)_{n, m} & =\hat{G}_{1, n-m}^{(k(\mu))}+i c(i \mu+i m) \hat{G}_{2, n-m}^{(k(\mu))}, \\
D(\mu)_{n, m} & =(i \mu+i m) \hat{G}_{3, n-m}^{(k(\mu))}, \\
S(\mu)_{n, m} & =\hat{F}_{3, n-m}, \quad V(\mu)_{n, m}=i \hat{G}_{2, n-m}^{(k(\mu))}, \quad \text { and } \\
\delta(n-m) & = \begin{cases}1 & \text { if } m=n \\
0 & \text { if } m \neq n\end{cases}
\end{aligned}
$$

We solve this generalized eigenvalue problem numerically by truncating the Fourier series representation from $\mathbb{Z}$ to $\{0, \pm 1, \pm 2, \ldots, \pm N\}$ for a discrete sampling for all possible $\mu$ and $\rho$ values. We note that due to the underlying symmetries in the problem, we may further restrict the $\mu$ interval from $\left[-\pi / L_{1}, \pi / L_{1}\right]$ to $\left[0, \pi / L_{1}\right]$. For traveling wave solutions of period $2 \pi, \mu \in[0, .5]$, while the parameter $\rho$ can be any number in $[0, \infty)$.

The convergence and reliability of this truncation was investigated by Curtis \& Deconinck CD10 as well as Johnson \& Zumbrun [JZ12. Those results do not formally apply to our setting, but can be easily generalized once we note that 
$S(\mu, \rho)$ is invertible. It follows that we can trust the numerical results obtained. With this truncation, we obtain $2(2 N+1)$ equations for $2(2 N+1)$ unknown Fourier coefficients $\hat{Q}_{n}$ and $\hat{N}_{n}$.

As seen in Figure 2, the adjustment to the maximal eigenvalue decays to $\epsilon_{\text {mach }}$ as $N$ increases. It should be noted that the decay rate for $h=.5$ is significantly slower than the decay rate for the other depths $h=1.5$ and $h=\infty$ with the same amplitude. This is not surprising: $a=.1$ corresponds to a significantly more nonlinear wave for $h=.5$ than for the other two depths.

We further establish confidence in our numerical approach by investigating its Cauchy convergence. Let $\lambda_{N}$ represent a particular eigenvalue corresponding to a truncation size $N$ (which corresponds to $2 N+1$ Fourier coefficients). We define the Cauchy error as

$$
e_{N}=\left|\lambda_{N}-\lambda_{N-1}\right|
$$

As we increase the size of our truncated matrix, the calculated eigenvalues should be found to converge numerically. We track the approximation of the eigenvalues with the largest real part as a function of truncation size for three different depths corresponding to traveling wave solutions with a dimensionless amplitude $a k=0.1$. The Cauchy errors are given in Table 1,

\section{Results}

4.1. Flat Water. Since we use a continuation approach, we start by carefully considering the stability of the trivial solution $\eta(x)=0, q(x)=0$, corresponding to a traveling wave of zero amplitude. We can analytically determine the spectrum of this trivial traveling wave. We choose the wave speed $c$ corresponding to the starting point of the bifurcation branch of periodic solutions to be

$$
c=\sqrt{\frac{g \tanh (h k)}{k}}
$$

where we choose $k=1$ for $2 \pi$ periodic solutions. Other bifurcation branches start elsewhere, but those branches correspond to mere scalings of the branch we consider. Their stability properties are identical, and no information is lost by considering only the one branch.

TABLE 1. Cauchy error for the calculation of the eigenvalue with largest real part for $h=.5, h=1.5$, and $h=\infty$ with $a=.1$.

\begin{tabular}{l|lll||r|rrr}
\hline$N$ & $h=.5$ & $h=1.5$ & $h=\infty$ & $N$ & $h=.5$ & $h=1.5$ & $h=\infty$ \\
\hline \hline 5 & $3.1 \mathrm{e}-03$ & $1.2 \mathrm{e}-07$ & $2.0 \mathrm{e}-08$ & 13 & $8.9 \mathrm{e}-09$ & $5.1 \mathrm{e}-16$ & $1.3 \mathrm{e}-15$ \\
6 & $3.2 \mathrm{e}-04$ & $3.0 \mathrm{e}-09$ & $3.3 \mathrm{e}-10$ & 14 & $2.0 \mathrm{e}-09$ & $5.7 \mathrm{e}-16$ & $2.1 \mathrm{e}-15$ \\
7 & $7.5 \mathrm{e}-05$ & $7.2 \mathrm{e}-11$ & $5.2 \mathrm{e}-12$ & 15 & $4.3 \mathrm{e}-10$ & $2.4 \mathrm{e}-15$ & $2.8 \mathrm{e}-15$ \\
8 & $1.8 \mathrm{e}-05$ & $1.7 \mathrm{e}-12$ & $7.9 \mathrm{e}-14$ & 16 & $9.3 \mathrm{e}-11$ & $3.5 \mathrm{e}-15$ & $3.0 \mathrm{e}-15$ \\
9 & $4.0 \mathrm{e}-06$ & $4.0 \mathrm{e}-14$ & $6.7 \mathrm{e}-16$ & 17 & $2.0 \mathrm{e}-11$ & $7.0 \mathrm{e}-16$ & $2.2 \mathrm{e}-15$ \\
10 & $8.7 \mathrm{e}-07$ & $1.2 \mathrm{e}-17$ & $6.8 \mathrm{e}-16$ & 18 & $4.3 \mathrm{e}-12$ & $3.4 \mathrm{e}-15$ & $4.0 \mathrm{e}-15$ \\
11 & $1.9 \mathrm{e}-07$ & $4.0 \mathrm{e}-16$ & $1.9 \mathrm{e}-16$ & 19 & $9.2 \mathrm{e}-13$ & $6.6 \mathrm{e}-16$ & $3.1 \mathrm{e}-15$ \\
12 & $4.1 \mathrm{e}-08$ & $6.4 \mathrm{e}-16$ & $1.2 \mathrm{e}-15$ & 20 & $1.9 \mathrm{e}-13$ & $1.2 \mathrm{e}-15$ & $8.1 \mathrm{e}-17$
\end{tabular}


Since the linear stability problem for this solution has constant coefficients, we easily determine the spectrum analytically. Repeating the method outlined in the previous section, we consider perturbations of the form

$$
\begin{aligned}
& \eta(x)=\epsilon e^{i \mu x+i \rho y+\lambda t} \sum_{j=-\infty}^{\infty} \hat{\eta}_{j} e^{i j x}, \\
& q(x)=\epsilon e^{i \mu x+i \rho y+\lambda t} \sum_{j=-\infty}^{\infty} \hat{q}_{j} e^{i j x},
\end{aligned}
$$

where $\epsilon$ is a small parameter.

Substituting this solution form into the equations of motion and simplifying as before, we find that the eigenvalues $\lambda$ are purely imaginary, as expected. They are given by

$$
\lambda_{m}^{s}=-i c(\mu+m)+i s \sqrt{g \kappa \tanh (\kappa h)}
$$

where $s= \pm 1, m \in \mathbb{Z}, \kappa=\sqrt{(\mu+m)^{2}+\rho^{2}}$, and $\kappa \neq 0$. Thus, for each perturbation (parameterized by $\mu$ and $\rho$ ), we can determine the growth-rates $\lambda_{m}^{s}$ for all $m \in \mathbb{Z}$.

Since all eigenvalues are purely imaginary, it follows that flat water is spectrally stable. Using the results of MacKay \& Saffman[MS86, we know that for an instability to arise in the water wave system, it is necessary for two eigenvalues with opposite signatures to collide, where the signature of a particular eigenvalue is given by

$$
\operatorname{sig}\left(\lambda_{m}^{s}\right)=-\operatorname{Im}\left(s \lambda_{m}^{s}\right) .
$$

Using the signature and the form of the eigenvalues $\lambda$, we see that a necessary condition for an instability to arise is that

$$
\lambda_{m}^{s_{1}}=\lambda_{n}^{s_{2}},-\operatorname{Im}\left(s_{1} \lambda_{m}^{s_{1}}\right)=\operatorname{Im}\left(s_{2} \lambda_{n}^{s_{2}}\right) .
$$

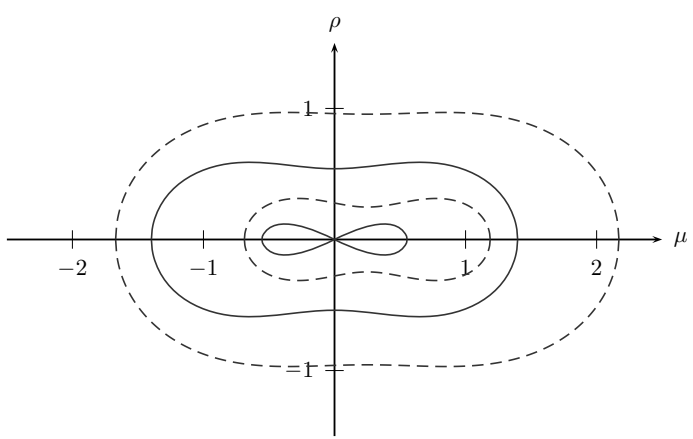

Figure 3. Resonant Curves for $h=.5$. The solid lines are associated with the Class I instabilities for $m=1$ and $m=2$, and the dashed lines with the Class II instabilities for $m=1$, and $m=2$. 
Solving these two equations, it follows that $s_{1}=-s_{2}$. This implies we can reduce the above two conditions to the single condition

$$
\lambda_{m}^{s}=\lambda_{n}^{-s}
$$

Following the work of McLean McL82a, McL82b and Francius \& Kharif FK06, there are two main conditions that we need to investigate for collisions of eigenvalues with opposite signatures:

Class I: $n=-m$ (the case of complex conjugates colliding at the origin),

Class II: $n=-m-1$ (collisions on the imaginary axis, away from the origin).

There are additional collisions of interest (such as when $n=-m-2$, etc). However, recalling that the perturbations are periodic in $\mu$ with period 1 , we can omit these additional collisions from consideration. These conditions give rise to the well-known resonant curves that are found throughout the literature (see for example (Ref. McL82a, McL82b, FK06]). They are shown in Figure 3 for $h=.5$. These curves connect the values of $\mu$ and $\rho$ for which eigenvalues with opposite signatures collide.

4.2. General Results. As mentioned in the introduction, much is known about the transverse stability of one-dimensional solutions of Euler's equations. Our intent is to demonstrate that the use of the AFM method reproduces these known results for the same parameter values as those used most recently by Francius \& Kharif [FK06], with less computational effort, or equivalently, with greater accuracy for comparable computational effort. The comparison is not straightforward. In [FK06], the authors used solutions that were allowed to have non-zero average value for a fixed depth whereas with our method, we strictly inforce zero-average values solutions.

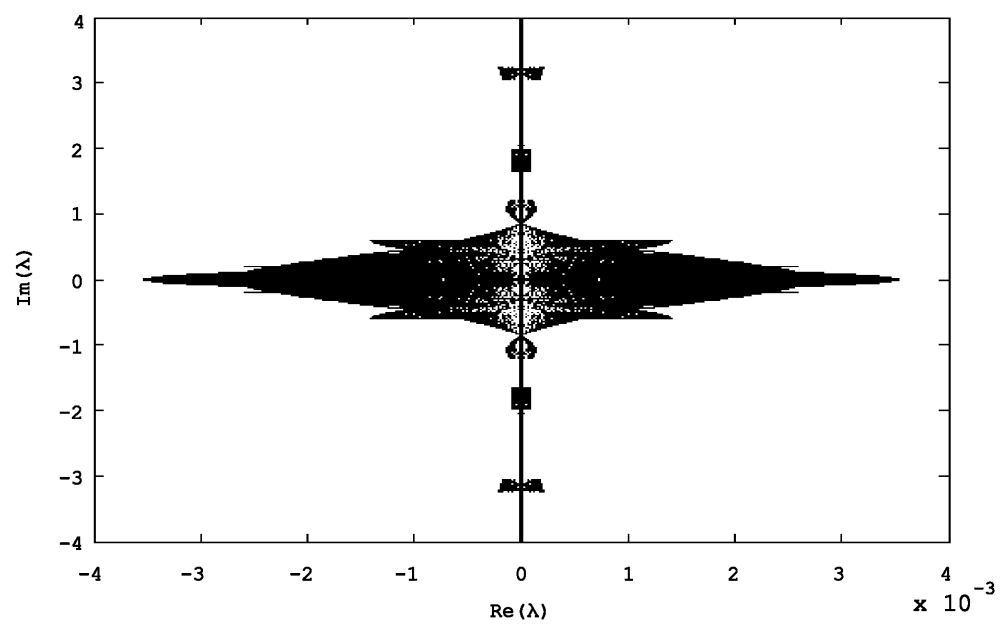

Figure 4. The spectrum $\{\lambda\}$ (real vs. imaginary part) for $h=.5$ and amplitude $a=.1$. Computed with $N=16$ Fourier modes, $1000 \mu$ values, and $300 \rho$ values. 


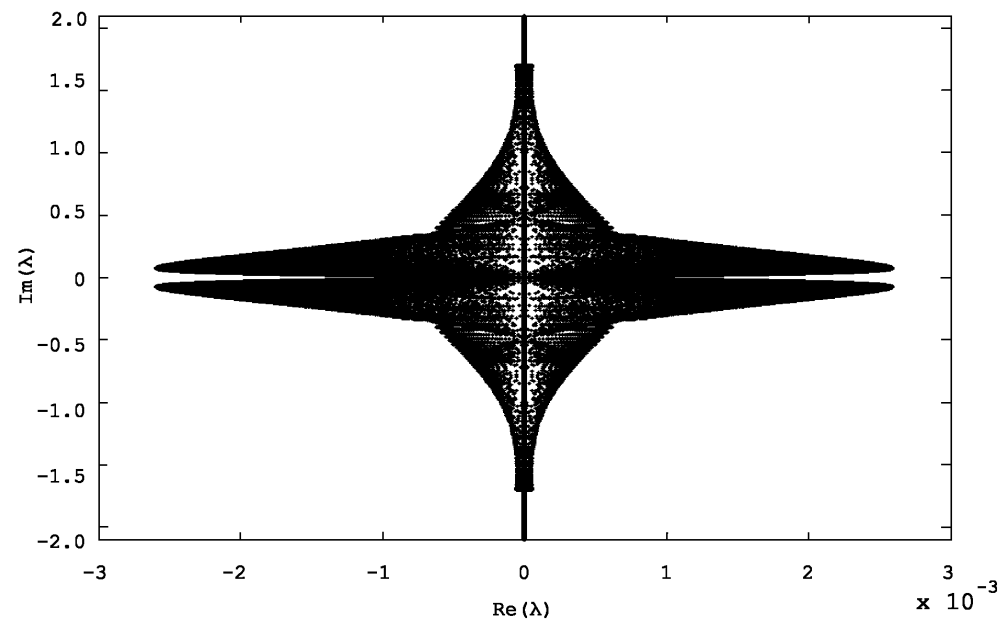

Figure 5. The spectrum $\{\lambda\}$ (real vs. imaginary part) for $h=1.5$ and amplitude $a=.1$. Computed with $N=16$ Fourier modes, $1000 \mu$ values, and $300 \rho$ values.

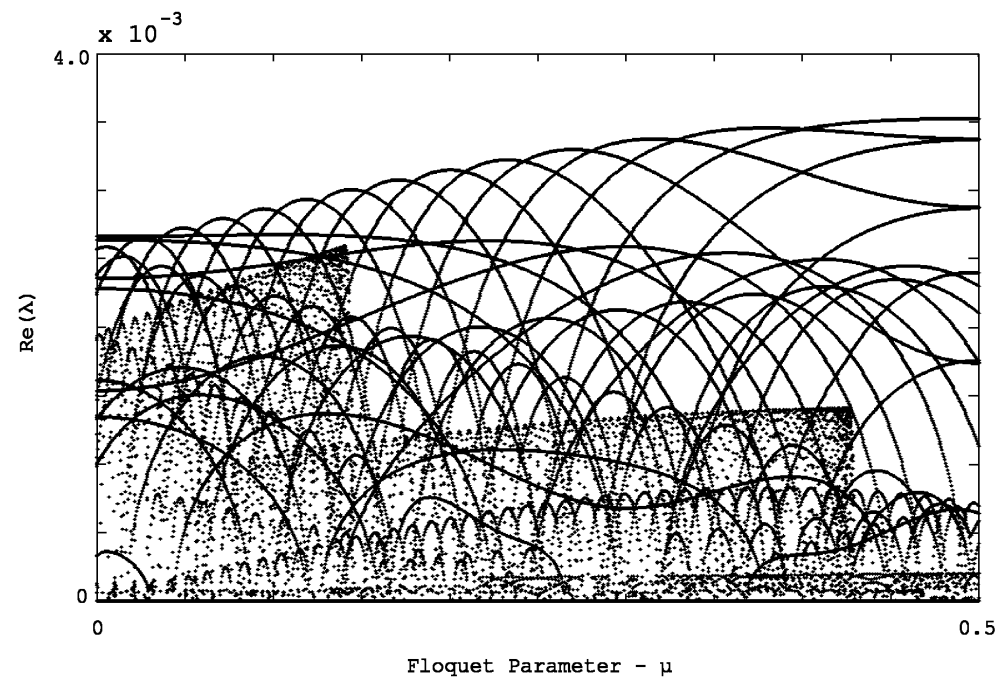

FiguRE 6. The real part of the spectrum as a function of the Floquet parameter $\mu$ for depth $h=.5$ and amplitude $a=.1$. Computed with $N=16$ Fourier modes, $1000 \mu$ values, and $300 \rho$ values. 


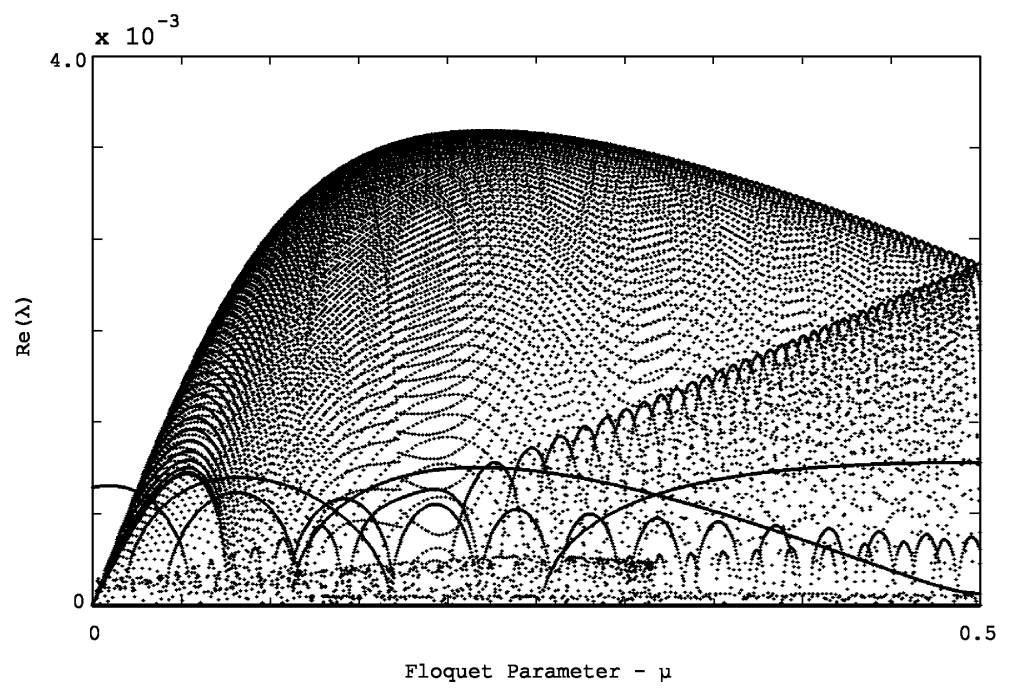

FiguRE 7 . The real part of the spectrum as a function of the Floquet parameter $\mu$ for depth $h=1.5$ and amplitude $a=.1$. Computed with $N=16$ Fourier modes, $1000 \mu$ values, and $300 \rho$ values.

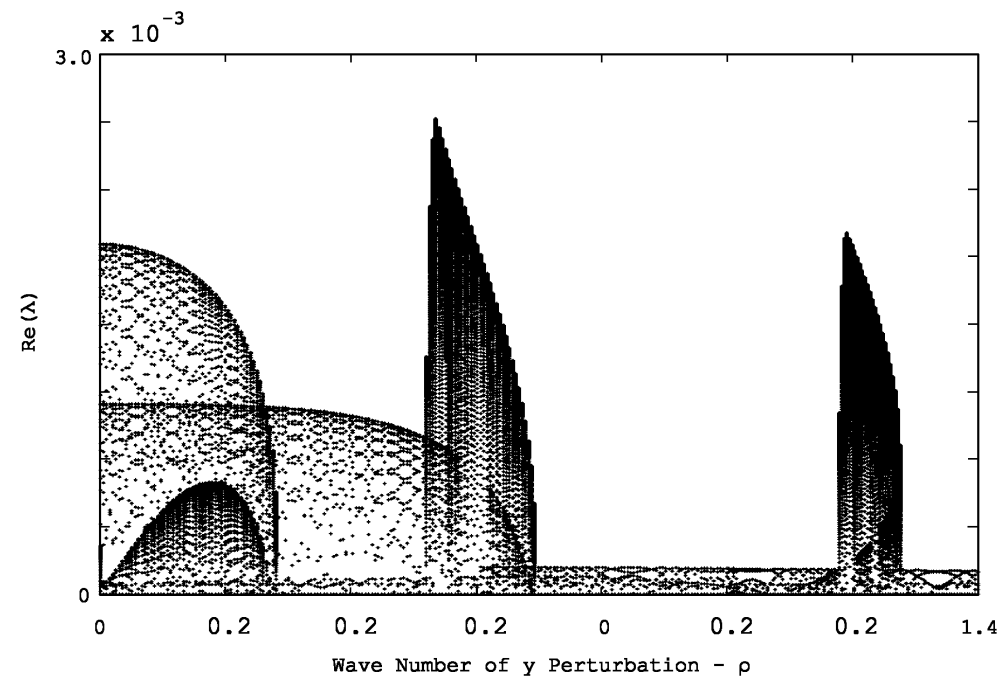

Figure 8. The real part of the spectrum as a function of the transverse wave number $\rho$ for depth $h=1.5$ and amplitude $a=.1$. Computed with $N=16$ Fourier modes, $1000 \mu$ values, and $300 \rho$ values. 


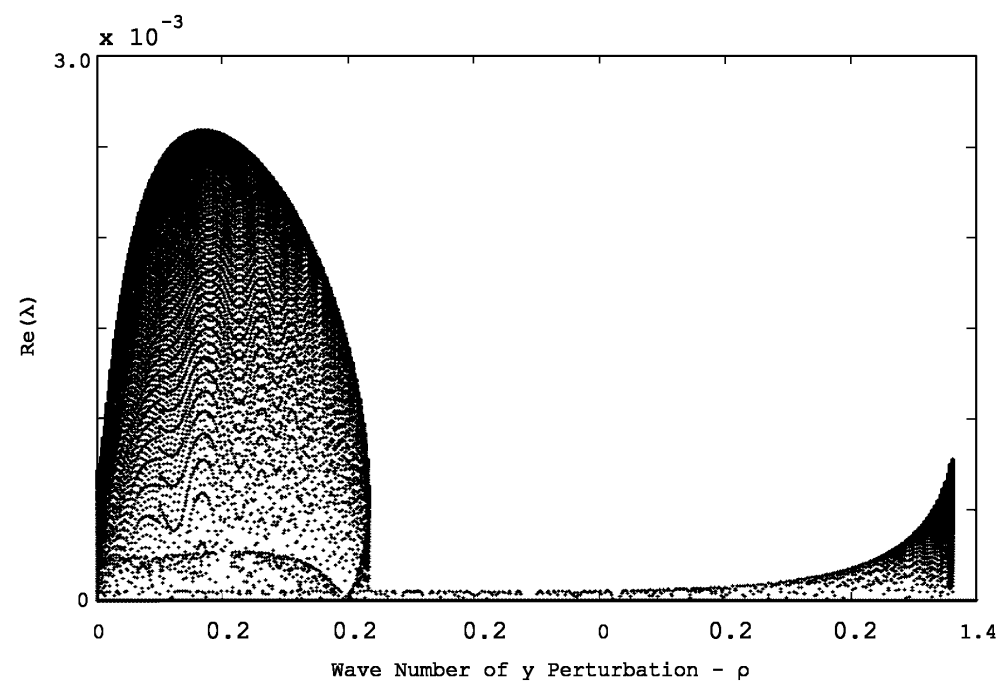

FiguRE 9. The real part of the spectrum as a function of the transverse wave number $\rho$ for depth $h=1.5$ and amplitude $a=.1$. Computed with $N=16$ Fourier modes, $1000 \mu$ values, and $300 \rho$ values.

We begin our investigations in shallow water $(h=.5)$ with a solution of moderate amplitude $(a=.1)$. We know that for this particular choice of wave height $a k=.1$ and depth $h=.5$ the wave is unstable with respect to one-dimensional perturbations [DO. Further, as expected, we find that this wave is unstable with respect to two-dimensional perturbations as demonstrated in the full spectrum given in Figure 4. Likewise, the full spectrum for $h=1.5$ is shown in Figure 5 . It is important to note that the largest real part of the spectrum in both cases is much larger than the largest real spectral elements when only one-dimensional perturbations are considered (see Ref. DO for comparison). This demonstrates that the dominant instabilities for these waves are the instabilities that arise from transverse or fully two-dimensional perturbations. However, this is not always the case. For $h=\infty$ and $a=.3$, we found that the dominant instabilities were the result of one-dimensional perturbations.

4.2.1. Maximum Growth Rates as a Function of $\mu$ and $\rho$. The full spectra demonstrated in Figures 4 and 5 provide a good visualization of the growth rates and frequencies present in the instabilities. We may also examine the unstable parts of the spectrum as a function of $\mu$ and $\rho$. First, we consider the spectrum as a function of $\mu$. In Figures 6 and 7 , we display the real part of the spectra for $h=.5$ and $h=1$. Additionally, we show the same spectra as a function of $\rho$ in Figures 8 and 9 .

It is important to note that as the values of the parameter $\rho$ are increased, bands of large instabilities appear (Figure 8 near $\rho=.6$ and $\rho=1.25$ ). As $\rho$ increases, these bands continue to appear. However, the magnitude of the dominant instability in these bands decreases with each successive band. Each of these bands corresponds to a specific resonant curve for the trivial solution, demonstrated in Figure 3 


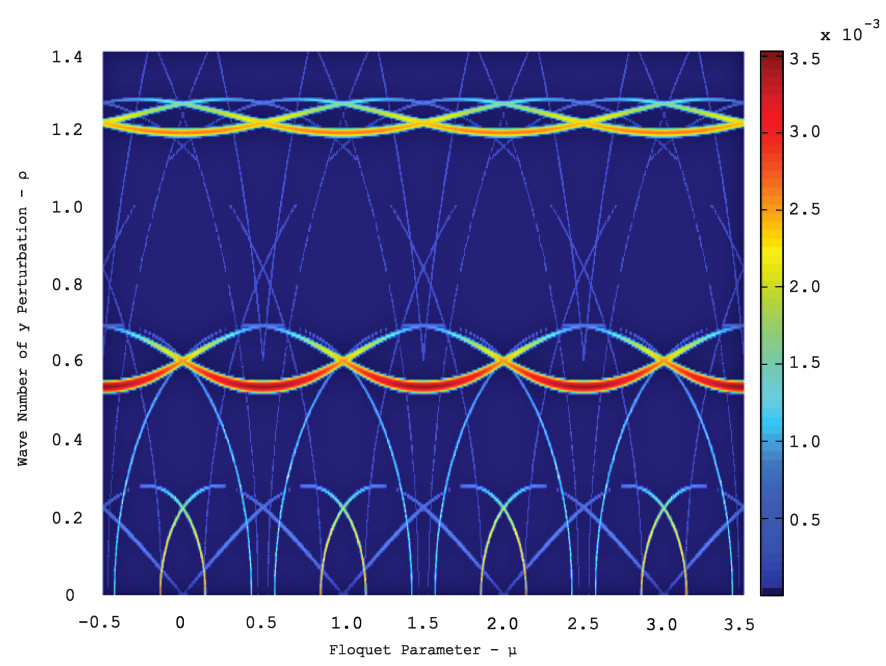

Figure 10. The maximum real part of the spectrum as a function of the Floquet parameter $\mu$ and the transverse wave number $\rho$ for depth $h=.5$ and amplitude $a=.1$. Computed with $N=16$ Fourier modes, $1000 \mu$ values, and $300 \rho$ values.

In addition, we visualize the instabilities in the $(\mu, \rho)$ plane where we use color to represent the magnitude of the maximum real part of the spectrum calculated for each perturbation. Figures 10 and 11 show the bands of instability. The periodicity with respect to the Floquet parameter $\mu$ is obvious in this representation. The instability bands given in Figure 10 again correspond to the resonant curves for the zero-amplitude solution. We notice the same trends as those found elsewhere

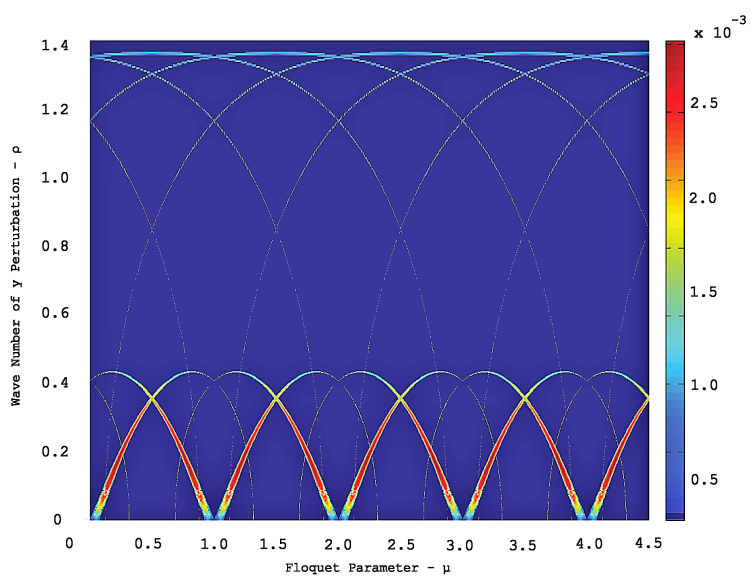

FigURE 11. The maximum real part of the spectrum as a function of the Floquet parameter $\mu$ and the transverse wave number $\rho$ for depth $h=1.5$ and amplitude $a=.1$. Computed with $N=16$ Fourier modes, $1000 \mu$ values, and $300 \rho$ values. 
in the literature FK06, McL82a, McL82b; as the amplitude of the underlying traveling wave increases, the instability bands (which start as curves) widen to unstable bands.

4.2.2. Eigenfunction Corresponding to the Most Unstable Eigenvalue. Yet another interesting investigation is to examine the eigenfunctions corresponding to the most unstable perturbations. These eigenfunctions provide a glimpse at the dynamics following the onset of instability, and allow one to predict the patterns that might be observed. For depths $h=.5$ and $h=1.5$, we calculate the sum of the eigenfunctions corresponding to the quadruplet of eigenvalues $\left(\lambda,-\lambda, \lambda^{*},-\lambda^{*}\right)$, where $\lambda$ is the eigenvalue with maximal real part, to incorporate the Hamiltonian structure of the stability problem. Combining the appropriate eigenfunctions and normalizing the amplitude to one, we obtain the level sets shown in Figures 12 and 13.

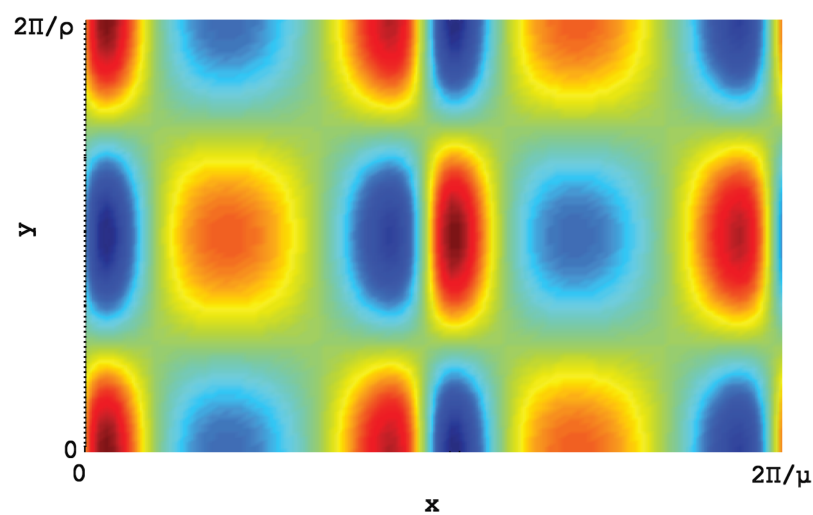

FiguRE 12. The combined eigenfunctions corresponding to the most unstable eigenvalue and its opposite, and their complex conjugates for depth $h=.5$ and amplitude $a=.1$.

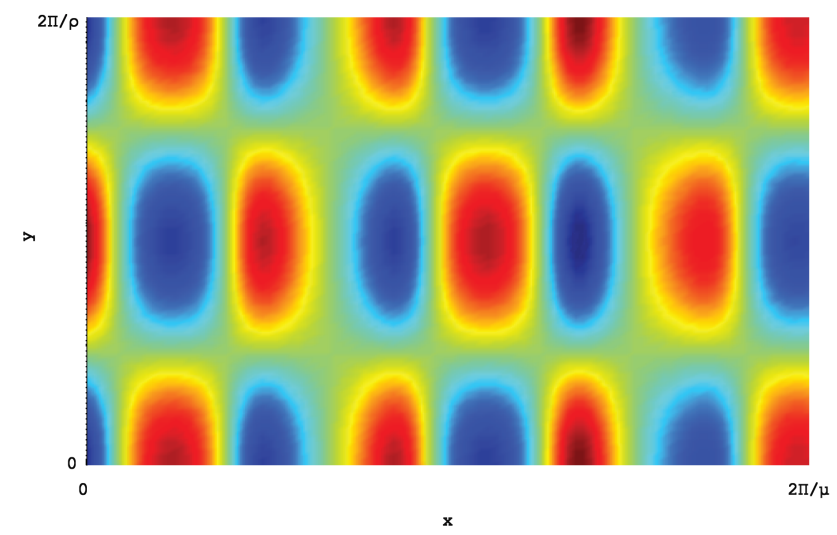

FIgURE 13. The combined eigenfunctions corresponding to the most unstable eigenvalue and its oppose, and their complex conjugates for depth $h=1.5$ and amplitude $a=.1$. 

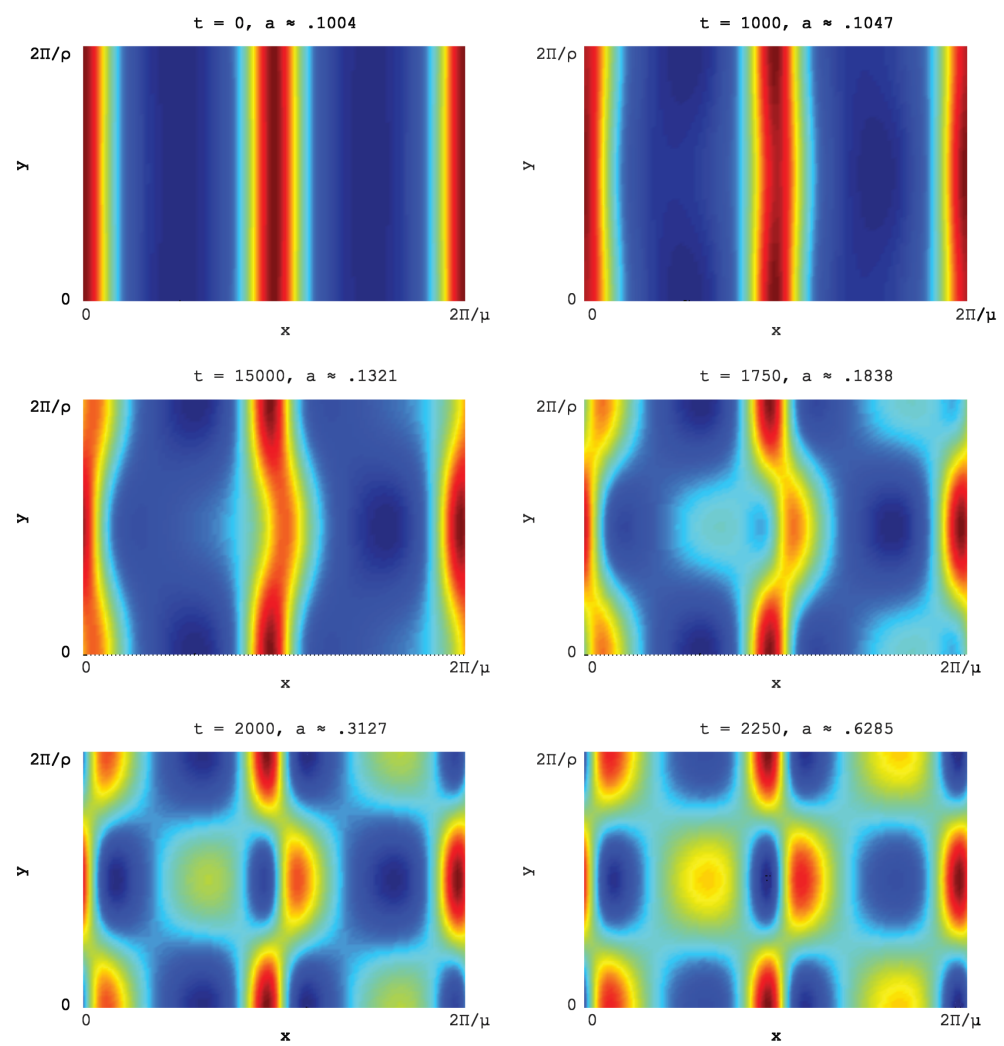

Figure 14. The solution to the linear problem for depth $h=.5$ and amplitude $a=.1$ corresponding to the most unstable perturbation. The dynamics displays the so-called snake instability, where wave crests are deformed but the amplitude is mostly unchanged along these deformed crests, at least initially. 

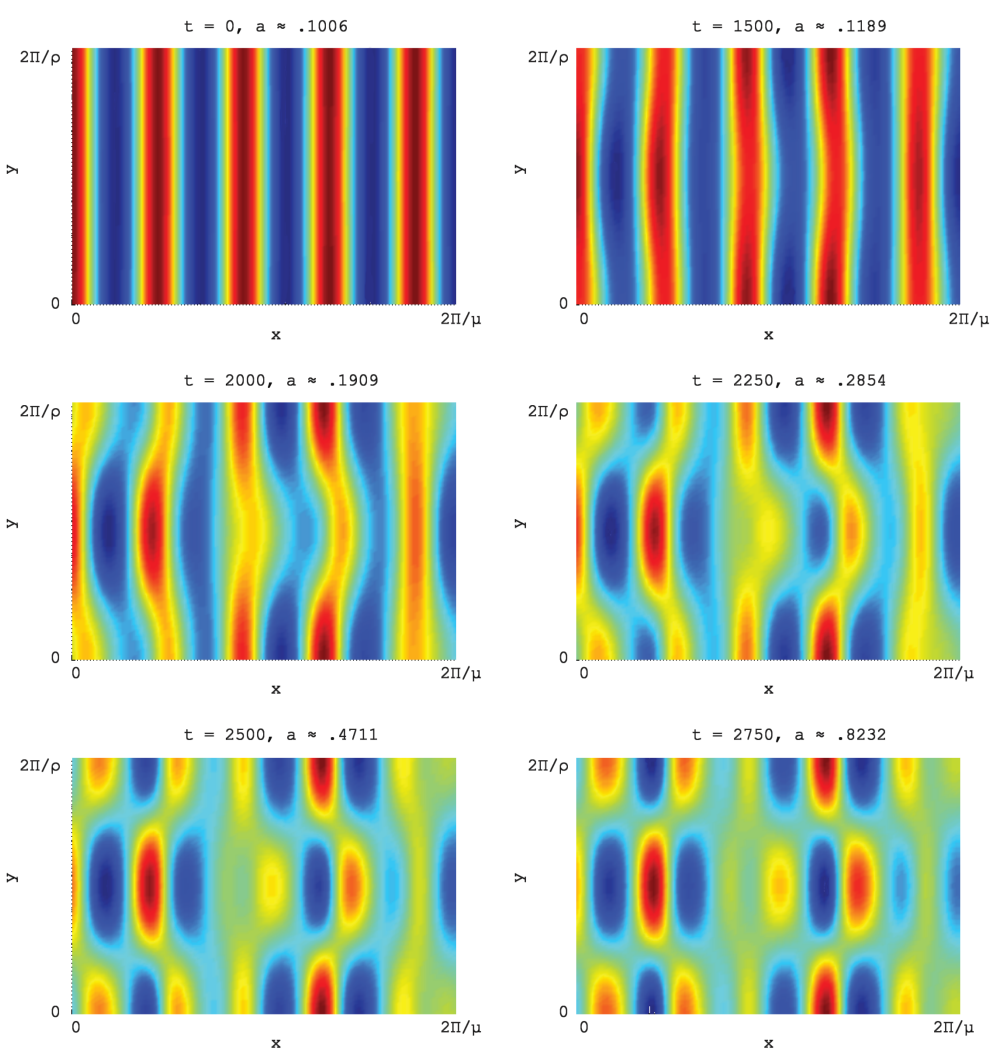

Figure 15. The solution to the linear problem for depth $h=1.5$ and amplitude $a=.1$ corresponding to the most unstable perturbation. The dynamics displays the so-called neck instability, where the amplitude along wave crests oscillates (most notably in the third frame), followed by a secondary snake instability. 
Recall that the perturbation of the one-dimensional solution is of the form

$$
\eta(x, y, t)=\eta_{0}(x)+\epsilon e^{\lambda t} e^{i \rho y} e^{i \mu x} \eta_{1}(x)+\text { c.c. }+\mathcal{O}\left(\epsilon^{2}\right)
$$

In addition to the mode corresponding to the complex conjugate eigenvalue, we also add those corresponding to the opposite eigenvalue and its complex conjugate. We use this to examine the linear time evolution of the traveling wave. For short times, before nonlinear effects become important, this demonstrates the dynamics of the perturbed wave pattern. Here "short time" should be considered relative to the size of the eigenvalue: the important measure is the product $\lambda t$. The time steps are shown in Figures 14 and 15. Since the perturbations are unstable, the amplitude of the solutions grow as demonstrated in each of the figures.

\section{Conclusions}

We have presented a small sampling of the instabilities of one-dimensional traveling wave solutions with respect to two-dimensional perturbations using the AFM formulation. By using the nonlocal formulation in combination with Hill's method, we have determined various spectra numerically with high degree of accuracy. Additionally, as demonstrated in Figure 2, the error of the approximation of the most unstable eigenvalue converges rapidly as a function of the number of Fourier modes used in the truncation.

When comparing our results to the method presented by Francius \& Kharif [FK06, our method converges for similar solutions using a smaller number of Fourier modes. To quantify this, where 80 Fourier modes are needed in [FK06] to obtain eigenvalues with Cauchy error on the order of $10^{-8}$, we only require 48 modes to obtain a comparable Cauchy error for the most unstable eigenvalue when $h=0.3$ and $a \approx 0.10$ (data obtained from the appendix of Ref. [FK06], available online only).

TABLE 2. Comparisons of Results with Francius \& Kharif for $h=$ 0.3 for convergence to six digits of accuracy. For the results from FK06, $a=.1$. However, for our results, $a=.1003$.

\begin{tabular}{c|c||c|c|}
\multicolumn{1}{c||}{} & \multicolumn{2}{c|}{ Francius \& Kharif $(a=.1)$} \\
Class Instability & Parameter Values & Modes & $\mathcal{R}(\lambda)_{\max }$ \\
\hline \hline Class I $(n=4)$ & $\mu=0, \rho=0.954$ & 60 & .034650 \\
Class I $(n=6)$ & $\mu=0, \rho=0.954$ & 70 & .041305 \\
Class II $(n=3)$ & $\mu=0, \rho=0.954$ & 70 & .021491 \\
Class II $(n=5)$ & $\mu=0, \rho=0.954$ & 70 & .040446
\end{tabular}

\begin{tabular}{c|c||c|c|}
\multicolumn{1}{c||}{} & \multicolumn{2}{c}{ Nonlocal $(a=.1003)$} \\
Class Instability & Parameter Values & Modes & $\mathcal{R}(\lambda)_{\max }$ \\
\hline \hline Class I $(n=4)$ & $\mu=0, \rho=0.954$ & 48 & .033527 \\
Class I $(n=6)$ & $\mu=0, \rho=0.954$ & 52 & .042458 \\
Class II $(n=3)$ & $\mu=0, \rho=0.954$ & 50 & .020000 \\
Class II $(n=5)$ & $\mu=0, \rho=0.954$ & 60 & .040528
\end{tabular}


TABLE 3. Comparisons of Results with Francius \& Kharif for $h=$ 0.5 for convergence to six digits of accuracy. For the results from FK06, $a=.17$. However, for our results, $a=.1696$.

\begin{tabular}{c|c||c|c|c|c}
\multicolumn{2}{c||}{} & \multicolumn{2}{c|}{ Francius \& Kharif } & \multicolumn{2}{c}{ Nonlocal Formulation } \\
Class Instability & Parameter Values & Modes & $\mathcal{R}(\lambda)_{\max }$ & Modes & $\mathcal{R}(\lambda)_{\max }$ \\
\hline \hline Class I $(n=4)$ & $\mu=0, \rho=1.102$ & 70 & .068502 & 48 & .073054 \\
Class I $(n=6)$ & $\mu=0, \rho=3.169$ & 70 & .056946 & 52 & .061713 \\
Class II $(n=3)$ & $\mu=0.5, \rho=0.475$ & 80 & .051826 & 48 & .053728 \\
Class II $(n=5)$ & $\mu=0.5, \rho=1.973$ & 80 & .067256 & 58 & .073044
\end{tabular}

In addition to the convergence rates, we also compared calculated growth rates with previously known results. Tables $2 \&$ 3, show comparisons of the calculated growth rates with the results from Francius \& Kharif[FK06] when $h=.3$ and $h=.5$. While there are some minor discrepencies between the final calculated growth rates for the chosen values of $(\mu, \rho)$, these discrepencies are easily explained since (1) the amplitudes of the traveling waves are slightly different, and (2) the average value of the traveling waves in Francius \& Kharif FK06 are nonzero, whereas the average value of the traveling waves using the nonlocal formulation are set to be zero. Furthermore, the results demonstrate the same patters of instabilities which were found by Francius \& Kharif FK06. For example, they showed that for $a=0.1$ and $h=0.3$ they dominant instability corresponded to a Class I instability (when $n=6$ ). Similarly, we found that the same trend exists for our calculations as well (see Table 2).

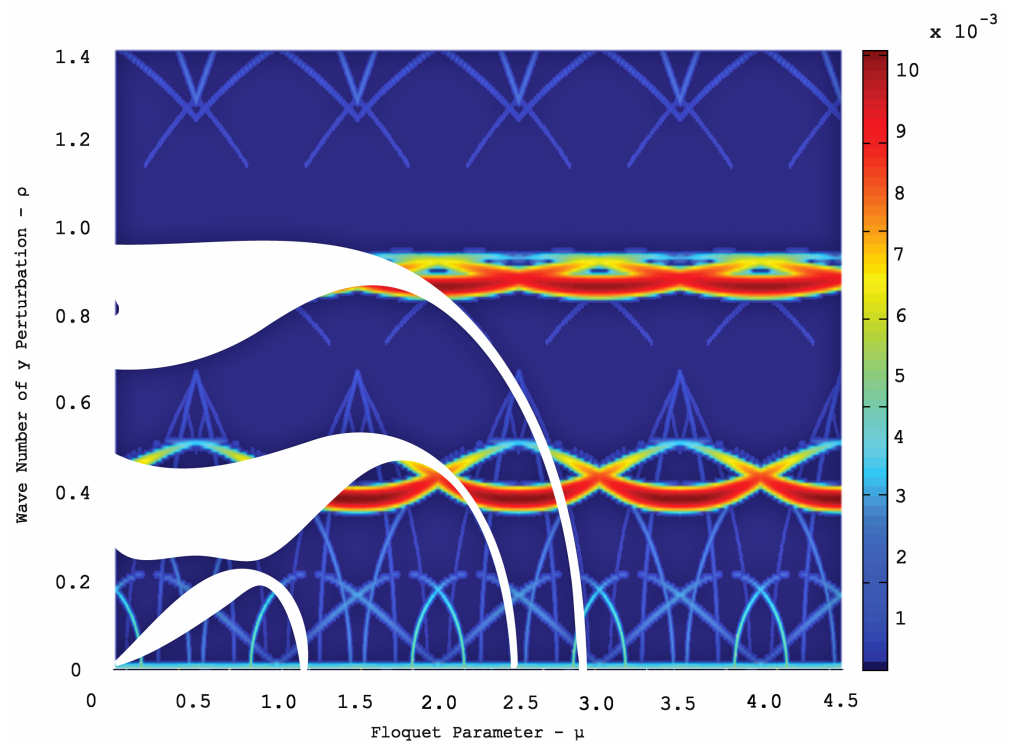

FiguRE 16. The maximum real part of the spectra as a function of Floquet parameter $\mu$ and wave number $\rho$ for $h=.5$ and amplitude $a=.160$ compared with the results obtained by Francius and Kharif[FK06] (outlined region). 
We have obtained results consistent with those of Francius \& Kharif. However, we have noticed that our instability regions (regions in the $(\mu, \rho)$ plane for which the spectrum intersects the right-half plane) appear to be smaller than those presented in FK06. For example, in Figure 16 we demonstrate the stability regions obtained in FK06 along with our own computed for $h=.5$ and $a=.160$. As shown in Figure 16, the general shape is comparable, but our instability regions appear to be smaller than those presented by Francius \& Kharif.

There are several possible explanations for the discrepancy in the size of the stability region. Perhaps the most logical is that we are able to calculate the underlying traveling wave solution to a high degree of accuracy using far fewer Fourier modes than those used in Francius \& Kharif FK06. This is a benefit of the reduced complexity of the nonlocal formulation for the one-dimensional traveling wave solutions, and perhaps its amenability to spectral numerical methods. In addition, It is important to note that since we incorporated the periodicity with respect to $\mu$ in our computations of the spectrum, our results are essentially tiled versions of the results presented in Francius \& Kharif FK06.

Summarizing, we have confirmed that for small amplitude waves, the dominant instability is two-dimensional (occurring when $\rho \neq 0$ ) with the magnitude of the dominant two-dimensional instability was sometimes multiple orders of magnitude greater than the dominant one-dimensional instability. The results were found earlier by Bryant Bry78. For $h=.5$, he found that for four-wave interactions, the dominant instability was fully two-dimensional. However, as the perturbations were time-evolved in his truncated equations, the two-dimensional instabilities dominated. As mentioned in Francius \& Kharif FK06, it would be interesting to explore the time evolution of a traveling wave perturbed with both of the dominant one- and two-dimensional instabilities to determine how each affects the long-time dynamics. This is an interesting possibility for future work since little is know about the time propagation of perturbations in shallow water using the fully nonlinear equations. Another interesting results is that for $h=.5$, the dominant instability corresponds to $\mu=1 / 2$, as also noted in the previous literature FK06. This implies that the dominant instability is phase-locked with the original solution expanded over twice the original period.

\section{References}

[AFM06] M. J. Ablowitz, A. S. Fokas, and Z. H. Musslimani, On a new non-local formulation of water waves, Journal of Fluid Mechanics 562 (2006), 313-343.

[AH08] M. J. Ablowitz and T. S. Haut, Spectral formulation of the two fluid Euler equations with a free interface and long wave reduction., Analysis and Applications 6 (2008), 323-348.

[Ben67] T.B. Benjamin, Instability of periodic wave trains in nonlinear dispersive systems., Proceedings, Royal Society of London, A 299 (1967), 59-79.

[BF67] T.B. Benjamin and J.E. Feir, The disintegration of wave trains on deep water. part i. theory., Journal of Fluid Mechanics 27 (1967), 417-430.

[Boh47] H. Bohr, Almost periodic functions, Chelsea Publishing Company, New York, N.Y., 1947.

[Bry78] P. J. Bryant, Oblique instability of periodic waves in shallow water, Journal of Fluid Mechanics 86 (1978), 783-792.

[CD10] C. W. Curtis and B. Deconinck, On the convergence of Hill's method, Mathematics of Computation 79 (2010), 169-187.

[CS93] W. Craig and C. Sulem, Numerical simulation of gravity waves, J. Comput. Phys. 108 (1993), no. 1, 73-83. 
[DK06] B. Deconinck and J. N. Kutz, Computing spectra of linear operators using Hill's method, Journal of Computational Physics 219 (2006), 296-321.

[DO] B. Deconinck and K. Oliveras, The instability of periodic surface gravity waves, J. Fluid Mech. Submitted.

[FK06] M. Francius and C. Kharif, Three-dimensional instabilities of periodic gravity waves in shallow water, Journal of Fluid Mechanics 561 (2006), 417-437.

[JZ12] Mathew A Johnson and Kevin Zumbrun, Convergence of hill's method for nonselfadjoint operators, SIAM Journal on Numerical Analysis 50 (2012), no. 1, 64-78.

[KR90] C. Kharif and A. Ramamonjiarisoa, On the stability of gravity waves on deep water, Journal of Fluid Mechanics 218 (1990), 163-170.

[LH78a] M. S. Longuet-Higgins, The instabilities of gravity waves of finite amplitude in deep water II. subharmonics, Proceedings, Royal Society of London, A 360 (1978), 489505.

[LH78b] M.S. Longuet-Higgins, The instabilities of gravity waves of finite amplitude in deep water I. superharmonics, Proceedings, Royal Society of London, A 360 (1978), 471488.

[McL82a] J. W. McLean, Instabilities of finite-amplitude gravity waves on water of finite depth, Journal of Fluid Mechanics 114 (1982), 331-341.

[McL82b] J. W. McLean, Instabilities of finite-amplitude water waves., Journal of Fluid Mechanics 114 (1982), 315-330.

$\left[\mathrm{MMM}^{+} 81\right]$ J. W. McLean, Y.C. Ma, D. U. Martin, P. G. Saffman, and H. C. Yuen, Threedimensional instability of finite-amplitude water waves, Physical Review Letters 46 (1981), no. 13, 817-820.

[MS86] R. S. MacKay and P.G. Saffman, Stability of water waves, Proceedings, Royal Society of London, A 406 (1986), 115-125.

[Nic09] D. P. Nicholls, Spectral data for travelling water waves: singularities and stability, Journal of Fluid Mechanics 625 (2009), 339-360.

[Whi67] G. B. Whitham, Non-linear dispersion of water waves, Journal of Fluid Mechanics 27 (1967), 399-412.

[Zak68] V. E. Zakharov, Stability of periodic waves of finite amplitude on the surface of a deep fluid., J. Appl. Mech. Tech. Phys. 9 (1968), 190-194.

Mathematics Department, Seattle University, Seattle, Washington 98122

E-mail address: oliveras@seattleu.edu

Department of Applied Mathematics, University of Washington, Seattle, WashingTON 98195

E-mail address: bernard@amath.washington.edu 